\title{
A VERY SINGULAR THEOREM
}

\section{An elusive idea that emerged on a pedestrian crossing revealed some of the mysteries inside black holes. Announced in barely a couple of pages, it has been worth the $\mathbf{2 0 2 0}$ Nobel Prize in Physics.}

I José M. M. Senovilla - DOI: https://doi.org/10.1051/epn/2021105

n Department of Physics at the University of the Basque Country (UPV/EHU)

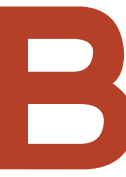

lack holes (BHs) are the most prudish objects in the Universe. Born in tremendous collapses of dying stars, eventually they close themselves off from the exterior, disconnecting from the outside world. External inquisitive observers are not given access, by any method, to their intimacies. $\mathrm{BHs}$ are sealed zones delimited by an inmaterial outer layer that cages everything there. Visitors are admitted, but not farewells: what gets into a $\mathrm{BH}$ is doomed to stay with these mysterious cosmic creatures.

The gravitational field of the mother star persists, though. BHs are so watchful about their secrets that, strictly speaking, what they allow us to observe is the final mass and spin of the progenitor just before it goes into isolation; if we are lucky, we can also perceive the stars and accretion disks that may orbit around them.

In 1964 Roger Penrose had a lucid yet fleeting thought. The idea emerged in the silence of a pedestrian crossing but, when he reached the other side, faded away. A diffuse feeling of elation remained that led him to review each moment of the day until, fortunately, the idea resurfaced [1]. The concept of trapped surface was born. He quickly scribbled the proof of a theorem that, in just two pages [2], uncovered internal properties of BHs. Fiftyfive years later, the theorem has been worth the Nobel Prize in physics. $\triangle$ Artist's impression of a black hole accretion disc. (C) ICRAR - ESA 


\section{They concluded that the neutron degeneracy pressure (due to Pauli's exclusion principle) is unable to maintain equilibrium for large masses. Gravity ends up prevailing if there is enough mass. 77}

\section{HORIZONS}

The $(x, c t)$ plane, with $c$ the speed of light. Nothing can travel faster than light, so no particle trajectory moving in the $x$-direction can exceed the $45^{\circ}$-slope with the vertical anywhere. This restriction defines, at each point, a future 'light cone', represented by green dashed lines for the points $\left(x_{1}, 0\right)$ and $\left(\mathrm{x}_{2}, 0\right)$. A particle $\mathrm{P}$ starts from rest at $\mathrm{t}=0$ to move with constant acceleration, so its speed is always increasing. Thus, $P$ describes a hyperbola - in red- with asymptote on the black, dashed, line. The P-trajectory crosses the $\left(x_{1}, 0\right)$ lightcone, but not that of $\left(x_{2}, 0\right)$. The border between these two situations is given by the asymptote line that acts as a horizon for $\mathrm{P}$ : no event above the horizon is ever observable by $\mathrm{P}$, not even if $P$ awaits infinite time. In fact, if $P$ watches the trajectory of $x_{1}$ at rest (blue dotted line), from P's point of view $\mathrm{X}_{1}$ tends to be "frozen" without ever reaching the horizon. The region above the horizon and the zone observable by $P$ are fully disconnected. A similar situation happens in black holes, which conceal themselves from the exterior by a cloaking horizon.

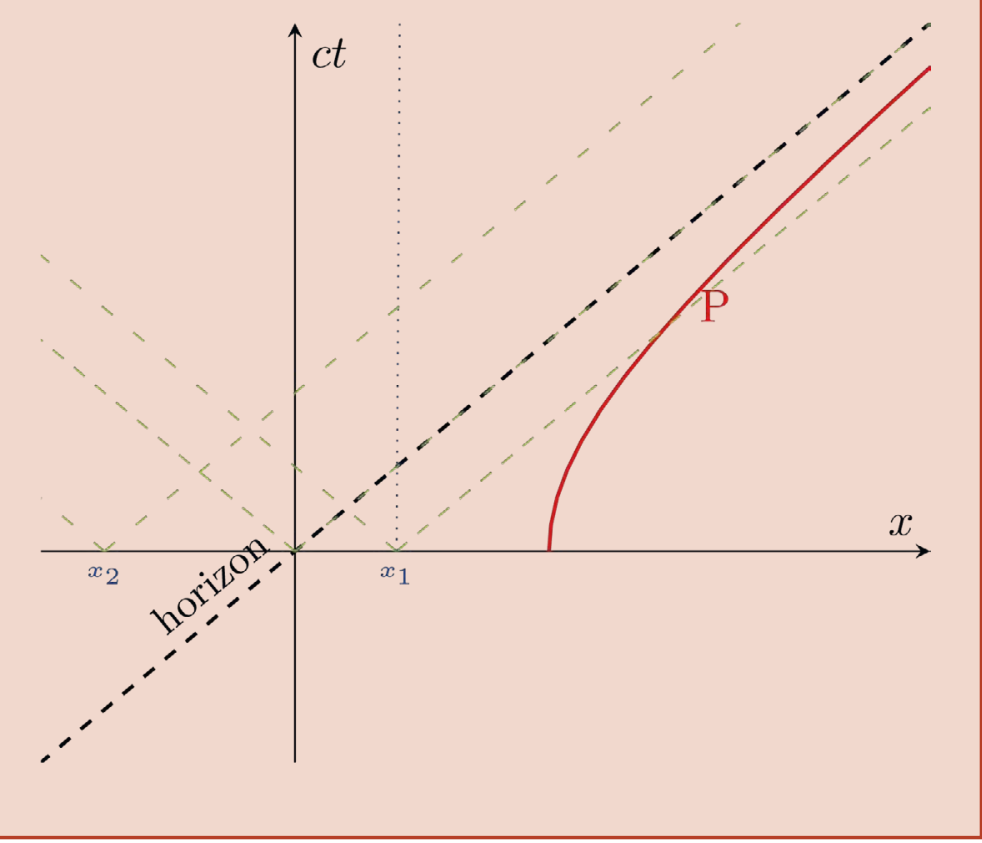

\section{Historical context}

In 1915 Einstein unified space, time, matter and geometry in the theory of general relativity (GR): the curved geometry of spacetime is a manifestation of the gravitational field created by matter and energy. Almost immediately K. Schwarzschild found the first exact solution of the GR field equations. It describes the gravitational field outside any spherically symmetric body and depends only on its mass $M$. The curvature was infinite at $r=0$, where $r$ is a variable such that the area of the spheres is $4 \pi r^{2}$. This singularity was somehow expected, as the Newtonian central field behaves as $\mathrm{M} / \mathrm{r}^{2}$. Yet, there was another problem at $r=r_{g}=2 \mathrm{GM} / \mathrm{c}^{2}-$ called gravitational radius - where $\mathrm{G}$ is the gravitational constant and $\mathrm{c}$ the speed of light in vacuum. Notice that $r_{g}$ is approximately $9 \mathrm{~mm}$ for the mass of the Earth, and $10^{-25} \mathrm{~m}$ for a person weighing $75 \mathrm{~kg}$. Schwarzschild concluded that such minuscule values of $r$ would be unattainable in realistic situations. However, in 1933 G. Lemaître detected a discrepancy with some $d y$ namical solutions found by A. Fridman, in which the area of spheres can be as small as desired. He showed that the supposed $\mathrm{r}_{\mathrm{g}}$-limit is fictitious and arises from having imposed a static solution: it can be eliminated by choosing coordinates that extend the spacetime to values of $r<r_{g}$, and $r=r_{g}$ simply describes a regular horizon. (Box.)

Still, are the dynamical regions with $\mathrm{r}<\mathrm{r}_{\mathrm{g}}$ physically accessible in reality?

This brings me to the subject of gravitational collapse. In 1939 Oppenheimer and Volkoff analyzed the stability of neutron stars - predicted a little earlier but only observed in 1967 by J. Bell. They concluded that the neutron degeneracy pressure (due to Pauli's exclusion principle) is unable to maintain equilibrium for large masses. Gravity ends up prevailing if there is enough mass.

The same year Oppenheimer and Snyder (O-S) studied the collapse of a spherical dust ball shrinking by its own gravitational attraction. They found that (i) the contraction is unstoppable proceeding to values of $r<r_{g}$ and eventually to the $r=0$ singularity, (ii) the star disconnects from any communication with the exterior so that (iii) surprisingly, outside observers never get to see the star crossing its gravitational radius, which thus becomes a horizon. The path to the black holes was thus cleared.

\section{The decade of marvels (1955-64)}

In 1955 A.K. Raychaudhuri published a fundamental formula that later became the basis of all singularity theorems. The Raychaudhuri equation describes the evolution of a pencil of world-lines in a gravitational field, allowing to prove that "caustics" generically develop: places where the curves intersect each other, analogous to the focus of a lens. This phenomenon is called the gravitational focusing effect. If the world-lines describe the motion of matter, a catastrophic singularity where the mass density becomes infinite is reached. 
In $1962 \mathrm{M}$. Schmidt discovered that the radio-source 3C 273 is extra-galactic. This seemed "unbelievable" because 3C 373 looks pointlike, its redshift implied a distance of 2.5 billion light-years yet it emitted a gigantic amount of energy outshining entire galaxies! Subsequent observations of these "quasi-stellar radio-sources" (quasars) accumulated. At that time, no process capable of producing such powers was known - not even nuclear reactions. Agitation increased in 1963 with the discovery by R. Kerr of the spacetime that (now) we know describes the gravitational field of a rotating $\mathrm{BH}$. It depends on the mass $\mathrm{M}$ and spin $\mathrm{J}$ of the $\mathrm{BH}$. It was quickly understood that an accretion disk around a rotating $\mathrm{BH}$ could convert matter into radiation with up to $42 \%$ efficiency —-truly amazing! In December of that year the "Texas Symposium on Relativistic Astrophysics" was devoted to quasars and GR [3].

J.A. Wheeler wanted to discern if singularities were actually an artifact of excessive idealizations, as in the O-S model that ignores the effects of pressure and rotation. He discussed it with Penrose. E.M. Lifshitz and I.M. Khalatnikov tackled the problem and concluded (erroneously) that singularities were just "problems of the coordinates". In December 1964 A. G. Doroshkevich, Ya. B. Zel'dovich and I.D. Novikov submitted a paper where they argued that irregularities of non-spherical stellar collapse were either eventually suppressed by physical processes or negligible. The O-S picture looked like a reliable description of stellar collapse.

To close the decade Penrose entered the scene. He was not convinced by the Lifshitz-Khalatnikov conclusions. When he was crossing the street inspiration enlightened him; [2] was also submitted in December.

\section{Penrose's theorem and its legacy}

Penrose proved that non-spherical irregularities were not capable of preventing the formation of singularities once a certain point of no return was reached. His theorem contained two revolutionary ideas, one of them brilliant, the other innovative.

The innovation was a clever way of avoiding the difficulties for a rigorous definition of singularities, because space-time itself breaks down at them. He decided to diagnose their existence if some hypothetical light rays (or other particle trajectories) come to a sudden end. If singularities were absent these paths would continue indefinitely. Their abrupt end, however, leaves the space-time incomplete. An unequivocal indication of the existence of a problem.

To characterise the "point of no return" in stellar collapse Penrose introduced the notion of trapped spheres, his genius idea from the crosswalk. As gravity is geometry, in dynamical situations geometrical quantities (such as area or volume) change with

\section{LASERS FOR QUANTUM TECHNOLOGIES}

$\mathrm{T}$ aking advantage of collective quantum effects has enabled the so-called first quantum revolution. Today, amid the second quantum revolution new sensing schemes offer higher sensitivities and better resolution thanks to the possibility to detect and control individual quantum states in microscopic systems like atoms, quantum dots, or color centers.

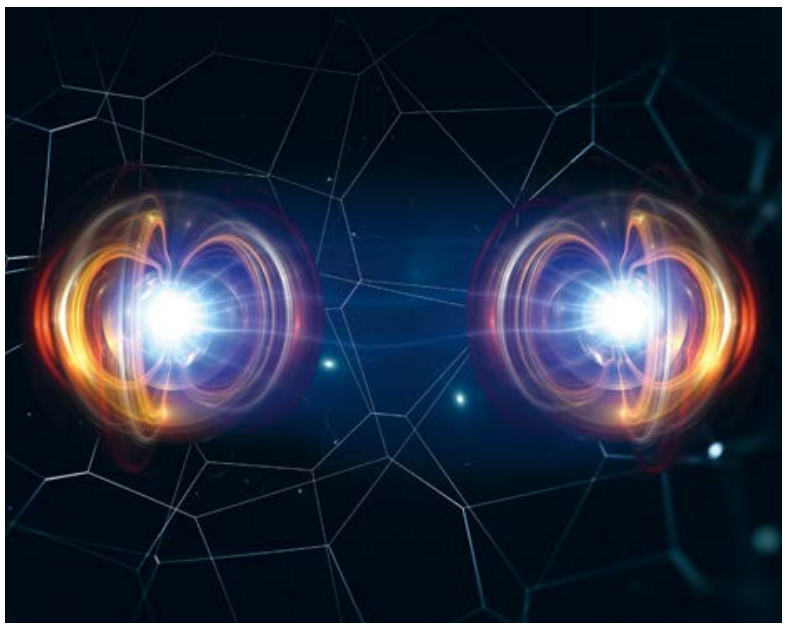

Read how the high performance lasers from HÜBNER Photonics are helping researchers in their quantum quest.

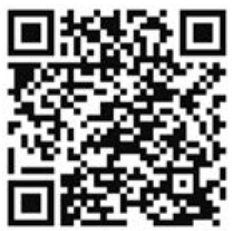

Scan the code to visit HÜBNER Photonics Quantum page (https://hubner-photonics.com/ applications/lasers-for-quantumtechnologies/)

\section{About the company}

HUBNER Photonics offer the full range of high performance Cobolt lasers, the CW tunable laser C-wave along, a full selection of C-FLEX laser combiners serving major instrument manufacturers and leading research labs for cutting-edge applications in the areas of fluorescence microscopy, flow cytometry, Raman spectroscopy, metrology, holography, nanophotonic and quantum research.

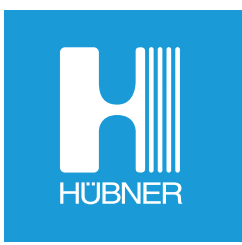


vFIG. 1: The concept of a trapped sphere. One dimension is suppressed. Time t goes up. A sphere $S$ at $t=t_{0}$ is represented by the circle, the area of $S$ by its length. An infinitesimal dt later flashes of light from $S$ reach two new spheroids: the circles in red and blue. In a stationary situation (left) the area of the red (blue) sphere is smaller (greater) than the area of S. If the world is contracting

(right), the blue circle may also have an area smaller than that of $S$ at $t_{0}$. Nothing can travel

faster than light, ergo such an $\mathrm{S}$ is said to be future trapped. time. In extreme cases, the area of some spheroids may decrease with time no matter how they evolve to the future (see Fig.1). In this sense, they are trapped. Penrose proved that, when they form, singularities usually develop. Trapped surfaces are stable against perturbations and they arise in the O-S collapse, hence they must also form in asymmetrical but basically spheroidal collapses regardless of the particular details of the star. In conclusion, singularities are not mere artifacts of coordinates or idealizations. It is also known [4] that no trapped sphere sticks out the $\mathrm{BH}$ horizon. Therefore Penrose's theorem reveals (some of) the secrets inside BHs .There (i) trapped spheres reside ergo (ii) space-time becomes incomplete. This is the GR prediction.

This result shook the relativistic community. Its impact was deep and stimulating. A plethora of "singularity theorems" [4,5] with diverse application, particularly to cosmology, were rapidly developed. The list of after-effects incited by [2] is rather extensive. [2] was a synthesis containing many of the concepts conceived by Penrose in the 1960's which ultimately resulted in the modern, post-Einstenian, era of GR $[5,6]$. In particular, he promoted the classical theory of $\mathrm{BHs}$ and devised a procedure to extract energy from them [7]. A refinement of this method, the Blandford - Znajek process, is the accepted explanation for the mechanisms powering quasars. As a conjecture (not proven yet), Penrose [7] proposed the existence of a "cosmic censor" in charge of surrounding all BH singularities with its cloak of concealment, its horizon, so that external observers are protected from any singularity-provoked hazards.

\section{Concluding remarks}

Gravitational configurations (planetary systems, stars, galaxies, clusters, ...) are free from singularities and effectively described by GR and its (post)-Newtonian limits. The only possible exceptions are the Universe and the interior of $\mathrm{BHs}$, due to the singularity theorems. In short, they provide supporting evidence that GR is an incomplete theory and must be (possibly quantum) corrected in extreme situations. Wonder is that the theory shows its own limitations! .

The 2020 Nobel Prize in Physics is well deserved Penrose's theorem has sheer beauty and important physical consequences. It spawned many fertile lines of research and uncovered intimacies of BHs that make them even more enigmatic. For more than twenty-five years stars have been observed at the centre of our galaxy orbiting something invisible, extremely compact and containing more than 4 million solar masses [6]. This hidden monster, most probably a $\mathrm{BH}$, is called Sagittarius $\mathrm{A}^{*}$. For this discovery Andrea Ghez and Reinhard Genzel have received the other half of the Nobel prize.

A splendid, spectacular, grand finale for the scientific treasure initiated by the 1965 mathematical theorem.

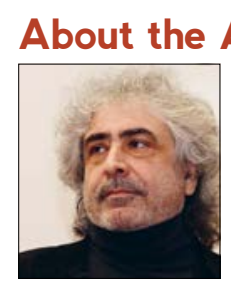

Author

Jose Senovilla is a Professor of theoretical physics in the Department of Physics at the University of the Basque Country (UPV/EHU) where he leads a research group on Gravitation and Cosmology.

\section{References}

[1] R. Penrose, The Emperor's new mind (Oxford Univ. Press, Oxford, 1989)

[2] R. Penrose, Phys. Rev. Lett. 14, 57 (1965)

[3] E. L. Schucking, Physics Today 42 8, 46 (1989)

[4] S. W. Hawking and G. F. R. Ellis, The large scale structure of spacetime, (Cambridge Univ. Press, Cambridge, 1973)

[5] J. M. M. Senovilla and D. Garfinkle, Class. Quantum Grav. 32 124008 (2015)

[6] Scientific Background on the Nobel Prize in Physics 2020: https://www.nobelprize.org/uploads/2020/10/advancedphysicsprize2020.pdf

[7] R. Penrose, Nuovo Cimento 1, 252 (1969).

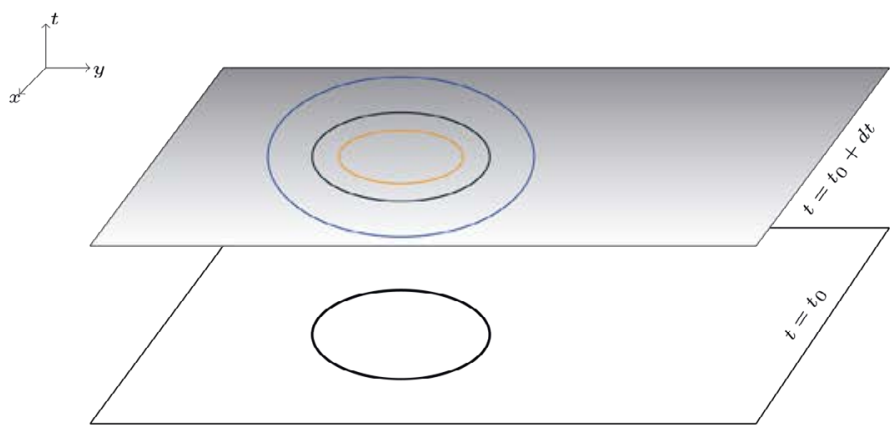

Stationary situation

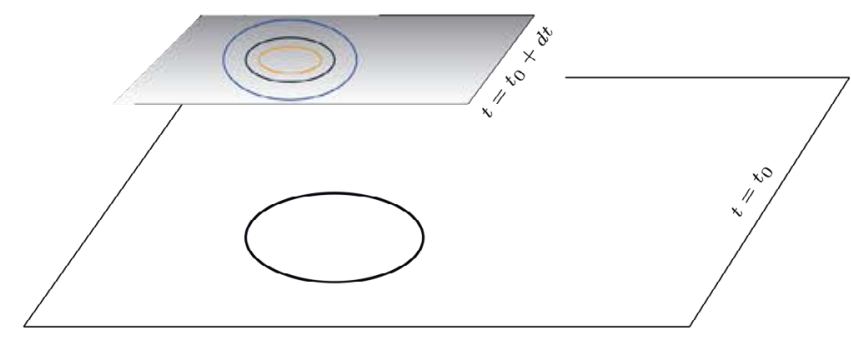

Spacetime in contraction 